\title{
OSTEOMYELITIS OF THE MAXILLA IN THE NEWLY-BORN
}

BY

\author{
H. D. WHITE, M.B., B.CH.
}

(From the Children's Department, St. Bartholomew's Hospital.)

Osteomyelitis of the maxilla, occurring in the newly-born and in babies up to the age of nine months, is a comparatively rare disease, but the condition has been described in medical literature from time to time, and thirty-six cases have been reported in all. The earliest was reported by Rees in $\mathbf{1 8 4 7}$ in a paper dealing with ' Uncommon forms of abscess in children '; in 1897 Sir D'Arcy Power reported a case under the heading, 'Empyema of the antrum in the newly-born'; other writers have described cases under the headings 'Phlegmon of the orbit,' and 'Premature eruption of teeth.' In 1904 Brown Kelly, of Edinburgh, observed a case, and collected together several others. He was probably the first to recognize the lesion as an osteomyelitis of the maxilla.

\section{Case reports.}

The typical signs and characteristic clinical picture produced by the disease are well illustrated in the two patients reported here.

Case 1.-.R. P., a male child, three weeks old, was brought to hospital because of a swelling in the mouth and a purulent discharge from the gum. There was also great difficulty in feeding the child.

Previous History. The child was born at full term, labour being normal. Birth weight was seven and a half pounds, and the child was breast fed. This was the mother's second pregnancy, the first terminating in a miscarriage at the fourth month. When ten days old the child was noticed to be very irritable, crying frequently, and would not take the breast. The right cheek was swollen and the right lower eyelid puffy. A pale swelling was noticed on the posterior part of the right upper alveolus. When fifteen days old the child was brought to hospital and a rudimentary tooth was removed from the right upper alveolus; this was followed by much purulent discharge from the cavity. Two days later a second rudimentary tooth was spontaneously discharged from the same cavity. The discharge of pus from the alveolus continued, and a purulent discharge from the right nostril followed. Feeding became more difficult and the child was admitted to hospital, being then twenty-four days old.

On Examination, the child was well nourished, and weighed eight pounds and two ounces. The temperature was $99.2^{\circ} \mathrm{F}$., pulse 
162 , and respiration 50. The right side of the face was swollen and oedematous, and the right eye was closed owing to puffiness of the right lower eyelid. There was a purulent discharge from the right nostril. In the mouth a swelling could be seen occupying the posterior part of the right upper alveolus extending outwards toward the cheek and inwards under the muco-periosteum of the hard palate. There was an opening on the gingival margin from which pus could be expressed, and a probe entered for half an inch, rough bone being felt. The swelling on the hard palate was soft and fluctuant. The pre-auricular and cervical glands were enlarged and tender. The heart and lungs were normal. The spleen could not be felt, and there were no other enlarged lymphatic glands. There was a small boil on the back which had been present for one week. The red blood count was 4,660,000 per c.mm., and the white cells 13,000 per c.mm., of which 82 per cent. were lymphocytes. Haemoglobin was 92 per cent.

Two days later a rudimentary tooth and a small fragment of bone were removed from the alveolar margin, and the facial swelling was beginning to subside. Pus continued to discharge into the mouth and from the nose.

When the baby was thirty days old an operation was decided upon. Thirty minims of paraldehyde in half an ounce of saline were given, and produced satisfactory anaesthesia. At the operation the edges of the cavity were avulsed and several small pieces of bone and one rudimentary tooth were removed; the cavity was plugged with adrenaline ribbon gauze, which was removed four hours later. The following day the child was feeding normally at the breast and from the bottle. The discharge of pus quickly dried up and the child made an uninterrupted recovery, leaving hospital three weeks after operation, having gained two and a half pounds in weight. The child has since done well, the alveolar margin has healed, and the face is not sunken. At the age of eight months he weighs nineteen pounds four ounces.

The cultures from the pus grew staphylococcus aureus.

Case 2.-R. W., a male child, aged two months, was brought to hospital because of a swelling of the right side of his face. He had been born prematurely at the seventh month, the mother having had influenza and pneumonia two weeks before delivery. There had been two previous pregnancies, the children being healthy.

When five weeks old the child became irritable. A swelling of the right upper alveolus was noticed, which discharged pus when the mother pressed it. A few days later swelling of the left upper alveolus was noticed and feeding became very difficult.

On Examination the child was well nourished, weighed nine pounds and one ounce. Temperature was $100^{\circ} \mathrm{F}$., pulse 152, and respiration 40. There was swelling and oedema of both cheeks and of both lower eyeids. In the mouth there was a tender swelling of the upper left alveolus and a sinus discharging pus in the right upper alveolus. The heart and lungs were normal and the spleen could not be felt. Two days after admission the child developed a swelling on the dorsum of the 
right hand, which rapidly spread up the arm to the shoulder and became fluctuant. Temperature rose to $104^{\circ} \mathrm{F}$., the pulse to 170 , and the respiration rate to 70 . The abscess was incised and much pus let out. Seventy c.c. of father's blood was given intravenously, but the child died two days later. Cultures of the pus grew staphylococci in large numbers, and some pneumococci. The Wassermann reaction was negative.

Post-Mortem Examination showed the changes associated with the pyaemic state. There was a purulent pericarditis and there were pyaemic abscesses in the heart muscle. There were small areas of consolidation in the lungs and many small pyaemic abscesses. The liver was pale and soft, the spleen large and soft, and the kidneys showed no changes. In the maxilla there was a small sinus in the right upper alveolus at the site of the unerupted second molar tooth, leading to a cavity lined by granulation tissue and communicating with the middle meatus of the nose. The tooth showed a rough, eroded area. There is no report on the state of the left side. There was an extensive necrosis of the right humerus at the diaphysis, and the periosteum had been stripped up for a considerable length as the result of a large sub-periosteal abscess.

\section{Aetiology.}

The disease affects newly-born children. It starts in the first three weeks of life in 90 per cent. of cases, but cases do occur up to the age of nine months. It is now generally accepted to be an osteomyelitis of the maxilla as the result of a blood-borne infection. An empyema of the antrum of Highmore is improbable as this is no more than a shallow groove at birth, measuring ten to twelve $\mathrm{mm}$. in its antero-posterior length and three to four $\mathrm{mm}$. in depth; the opening is along the whole of the lower shelf of the groove. Infection of a dental sac has been suggested as the cause, but it is difficult to decide whether this is primary or secondary. In nearly all the cases reported there was involvement of one or more dental sacs. If these were the primary sites of infection one would expect to find some cases in which the disease process had remained localized. In the majority of cases reported symptoms have pointed to an involvement of the whole bone. This latter fact is strongly in favour of the infection being a true osteomyelitis, with secondary involvement of the dental sacs.

There are many predisposing causes, and among these trauma during birth plays a prominent part. The maxilla at birth is the site of great vascular activity and, being a fixed bone, is very liable to trauma during an abnormal labour. Three cases are recorded following occipito-posterior presentation and three after forceps delivery. Infection of the nasal mucous membrane during passage down the birth canal or injury to the mouth after delivery may be the source of entry for the infection. Cases have been reported associated with areolar infection, mammary abscess, boils, and other sources of focal infection. 


\section{Pathology.}

In only nine of thirty-six cases is bacteriological evidence available, and in these staphylococcus aureus was present in seven. Streptococci were found in one case and in another tubercle bacilli. French authors have cited syphilis as the cause, but in no case has the Wassermann reaction been positive.

The local lesion in the maxilla probably starts in a blood vessel, either as a direct infection or as a result of a septic embolus. A thrombophlebitis is set up and the vessel becomes occluded, leading to necrosis of the distal parts. Wilensky has made a study of the blood supply of the maxilla, and he regards the slight variations in symptoms as evidence that the initial process has started in one or other of the many branches and vascular loops of the internal maxillary artery. This is substantiated by the cases recorded in the literature, in one of which the entire bone was involved, representing the main trunk artery, while in others the process was localized to the alveolar and nasal branches.

In this connection it may be remembered that the centre of ossification of the maxilla is situated just above the canine and first molar tooth buds. This region is therefore especially vulnerable.

As a result of the necrosis, bony sequestra are frequently formed. If the blood supply of a dental sac is cut off, the rudimentary tooth behaves as a sequestrum, and milk teeth acquire their crown at about the time of the child's birth. This fact helps to establish the time of onset of the disorder when teeth are shed. Following the expulsion of all bony sequestra, healing takes place. Owing to the small amount of periosteum on the maxilla and to its poor nourishment from outside, this process is slow, and new bone formation is frequently incomplete. Locally the disease may progress, causing a secondary nasal infection, a periostitis or a sub-periosteal abscess of the orbit, or a spread may take place through the cribriform plate of the ethmoid, leading to fronta] abscess formation. The infection may reach the general circulation leading to pyaemia and the formation of metastatic abscesses. Such abscesses have been recorded in the hand, the shoulder, the pericardium, the heart-wall, the lungs, and the kidneys. In one case reported here a secondary suppurative periostitis of the humerus resulted. Bronchopneumonia may complicate the picture arising as a blood-borne phenomenon, or from aspiration.

Signs and symptoms.

The onset of the disease is mild, there being a few days during which the child is irritable, cries frequently, refuses the breast or bottle, and is generally difficult in management. Fever is not a predominant symptom during this or any other stage of the disease. In only four of the cases reported was pyrexia marked, and in one of these convulsions were observed. Following this prodromal stage, symptoms appear by which the disease may be referred to the maxilla, and they may present at any, or all, of its aspects, namely, orbital, facial, alveolar and palatine, or nasal. 
The most common onset is swelling of the face on the efiected side with oedema of the eyelid. This was the initial symptom in 70 per cent of cases. Of the remaining cases, the initial symptom in 20 per cent. was swelling of the palate or alveolus, in 9 per cent. profuse nasal discharge, and in one case conjunctivitis and exophthalmos. With the spread of the disease the triad of symptoms, swelling of face and eyelids, discharge from the nostril, and swelling and discharge from the alveolar margin, gives the characteristic clinical picture.

When orbital and facial symptoms predominate there is swelling of the cheek and eyelids, which may cause complete closure of the eyes. With free drainage of the pus the swelling may subside, but in other cases it progresses to abscess formation; such abscesses tend to point at the outer and inferior border of the outer canthus, leaving fistulae, which usually heal after the discharge of dead bone. Conjunctivitis and chemosis are frequently present. Exophthalmos is described in 20 per cent. of cases recorded. It varies from a mild degree to a fairly severe proptosis. When it occurs it is evidence of the spread of the disease to the orbital plate of the maxilla, and is almost certainly caused by a sub-periosteal abscess in the orbit. In no case has the eye itself been affested. Retrobulbar neuritis and optic atrophy do not occur as in ethmoid infection in adults. Glandular enlargement is not mentioned in the cases reviewed, but in one case recorded in this paper the submaxillary and pre-auricular lymphatic glands were enlarged. Retropharyngeal abscess does not occur. Pressure on the swollen face frequently produces an augmented flow of pus from the nostril and alveolar margin, and in one case egg-shell crackling was recorded.

Nasal discharge is the only nasal sign; it may occur at the onset of the disease or during the spread of the infection; it is, as a rule, unilateral, small in amount at onset, becoming profuse as the disease progresses, and it has been recorded as the only residual sign several months after the disease has ceased to be active.

Swelling of the alveolar margin in the region of the canine or first molar tooth is a constant early sign. The swelling may extend on to the hard palate; it is soft and fluctuant, unless it has already burst, leaving an alveolar fistula, through which purulent matter discharges. A rudimentary tooth may, be seen as a white plaque on the gums in the region of the swelling. The spontaneous eruption and discharge of such teeth is recorded in the majority of cases, and probably occurs in all cases of the disease. Sequestra are commonly discharged through the alveolar margin; in two cases, however, small pieces of dead bone were extruded from a fistulous opening on the cheek. A probe introduced into an alveolar fistula, enters the dental sac, and frequently communicates with the nose, the thin plate of bone which separates antrum and dental sac being rapidly broken down by the disease process.

The clinical picture is so clear that other aids to diagnosis are hardly necessary, and brief mention only will be made of them. Transillumination has been attempted, but it is unsatisfactory in children. Skiagrams 
of the maxillary region are of use in determining the extent of the disease and the presence of sequestra. A mottled appearance is present on the affected side.

\section{Complications.}

It would appear from the cases recorded in the literature that the following complications may occur during the course of the disease :septicaemia (one case with a positive blood culture); pyaemia, with multiple metastatic abscesses in the lungs, kidneys, etc. (two cases); gastro-enteritis; parotitis (one case); cerebral abscess resulting from a direct spread of the disease through the cribriform plate of the ethmoid (one case of frontal abscess); aspiration pneumonia; orbital periostitis and sub-periosteal abscess.

\section{Treatment.}

The main objects of treatment are to prevent complications and to attempt to stop the spread of the infection by ensuring free drainage of pus and dead tissues.

The mouth should be cleansed hourly, a solution of 1 in 8,000 permanganate of potash being a suitable disinfectant. Sinuses and cavities in the alveolus should be irrigated with normal saline, and the orifice should be kept open until the discharge of pus has ceased. The child should be nursed on the affected side and, if possible, almost on the face, thus minimizing the risk of aspiration of the pus.

Breast or bottle feeds are not contra-indicated, but should much difficulty arise, gavage feeds can be instituted. It is important to keep the child sufficiently nourished.

The indications for operative measures are:-(1) The presence of abscesses; (2) the progress of the disease, and persistence of purulent discharge; (3) the presence of sequestra and necrosed rudimentary teeth. Abscesses commonly appear on the face or alveolar margin. The latter should be incised as soon as fluctuation is detected; a rudimentary tooth is frequently dislodged during this procedure, which may be followed immediately by a regression of the symptoms after the free discharge of pus. An abscess on the face should be opened early, as damage to the eye may result from delay. Of the cases reviewed, twenty-four were operated on, and in five death occurred. The operations performed varied from the removal of teeth and sequestra from the alveolus to extensive operations on the maxilla. Of the twelve patients not operated on, four died. Wilensky advises operative measures to be as conservative as possible, as extensive operations in acute cases may spread the infection. Exploration of the maxilla by the alveolar route is probably the operation of choice, and this was done in one of the cases reported in this paper. An incision is made into the alveolus on its outer aspect, and the dental sac explored. Rudimentary teeth and dead bone are removed. In most cases a communication between the nose and 
mouth will be established, and drainage will be adequate. The cavity should be plugged and the plug removed the following day, when irrigations may be resumed. No attempt should be made to preserve teeth or tooth buds, as these may be responsible for maintaining the infection. In the cases reviewed the majority of operations performed were of this nature, but, in some cases, more extensive explorations were made by a facial route. This does not appear to be a favourable mode of approach, as it must result in permanent disfigurement in later life, while the drainage through such an incision may be hindered by mechanical difficulties.

No mention is made in the cases reviewed of the anaesthetic used. In one of the cases now reported, thirty minims of paraldehyde given in saline per rectum provided adequate anaesthesia.

\section{Prognosis.}

The disease must be considered as a serious illness. In the cases reviewed the mortality rate was 26 per cent. The disease may last a few weeks or several months. The formation of sequestra and their gradual extrusion is responsible for delayed healing in many cases, but when these are discharged spontaneously or removed at operation, rapid recovery follows. Future health is in no way impaired. In two cases the face remained sunken on the affected side for some time afterwards, but the disfigurement was only slight. Otherwise the absence of the teeth which have been lost or removed, is the only unpleasant late result.

\section{Summary.}

1. Osteomyelitis of the maxilla in the newly-born is a bacterial infection, usually staphylococcal or streptococcal, affecting healthy children between the ages of two weeks and nine months.

2. It is a haematogenous infection localizing in the maxilla, either in the bone itself, in the region of the centre of ossification, or in a tooth bud.

3. The pathology is the same as that for osteomyelitis in older children.

4. The symptoms are clear cut and easily recognized.

5. Some operative measure is indicated in the majority of cases, and should be instituted early.

6. The prognosis is in the first place a serious one, but if improvement follows operation, then a more favourable prognosis may be given.

7. Residual symptoms are few, and a complete recovery is usual.

I am indebted to Dr. Charles Harris and Prof, G. E, Gask for permission to publish these two cases. 


\section{REFERENCES.}

Bass, M. H., Am. J. Dis Child., Chicago, 1928, XXV, 65.

Brown-Kelly, A., Edinburgh Med. J., Edinb., 1904, XVI, 302.

Collet, F. J., Arch. Int. de Laryng., Paris, 1922, I, 1041.

Douglas, A., Brit. Med. J., Lond., 1898, i, 368.

Dujardin, A., J. d. sc. méd. de Lille, 1888, XII, 169 (abst. Ann. occul., Paris, 1888, XCIX, 194).

Francois, M., Thèse de Paris, Paris, 1914.

Hughes, E. E., Brit. J. Child. Dis., Lond., 1921, XVIII, 89.

Marx, E., Brit. J. Ophthal., Lond., 1922, VI, 25.

Mayer, E., Med. Rec., New York, 1901,

Moure, E. J., Rev. Laryng., Paris, 1896, II, 1265.

Power, D'A., Brit. Med. J., Lond., 1897, ii, 808.

Rees, G. A., Lond. Med. Gaz., Lond., 1847, IV, 850.

Roehm, H. E., Am. J. Dis. Child., Chicago, 1931, XLII, 1171.

Ropke, D., Arch. Otology., New York, 1899, XLV, 1433.

Rudaux, M., Annals de Mal d'Oreille, 1895, II, 289.

Wilensky, A. O., Arch. Surg., Chicago, 1932, XXV, 183.

Idem, Am. J. Dis. Child., Chicago, 1932, XLIII, 431.

Wolff, I. J., Am. J. Dis. Child., Chicago, 1933, XLV, 1040.

Wood, F. C., Brit. Med. J., Lond., 1897, ii, 1259. 